\title{
PARTITION CODING USING MULTI-GRID CHAIN CODE AND MOTION COMPENSATION
}

\author{
Ferran Marqués and Antoni Gasull \\ Dept. of Signal Theory and Communications \\ ETSETB - Universitat Politècnica de Catalunya \\ Campus Nord - Módulo D5 \\ C/ Gran Capità, 08034 Barcelona, Spain \\ Tel: (343) 40164 50, Fax: (343) 4016447 \\ E-mail: ferran@gps.tsc.upc.es
}

\begin{abstract}
In this paper, a lossy partition coding technique is presented which leads to decoded partitions with unnoticeable losses. It uses an hexagonal grid for contour representation and it is based on the concept of Multi-Grid Chain Code. This coding technique can be used in intra-frame mode or, in combination with partition prediction techniques, in interframe mode. In intra-frame mode, it leads to an average saving of $25 \%$ of the coding cost with respect to Chain Code techniques. The savings on inter-frame mode depend on the motion prediction approach. Results coding binary shapes (concept of VOP) as well as partitions with an arbitrary set of regions are presented.
\end{abstract}

\section{INTRODUCTION}

Among the different coding methods grouped under the name of second generation coding techniques [5], there is an increasing interest in segmentation-based image sequence coding approaches. This interest is due to the fact that these techniques have a strong potential for increasing the coding efficiency as well as an inherent capability for handling contentbased functionalities [3].

The segmentation procedure should yield partitions whose regions are homogeneous in some sense (e.g.: spatial or motion homogeneity). Due to this homogeneity, the information of each region can be separately coded in an efficient manner. This homogeneity has to be ensured since, with respect to classical coding techniques, segmentation-based approaches must code an additional information which is the contours; that is, the partition sequence. This partition information has a rather high coding cost and, therefore, efficient partition coding techniques are necessary.

The same problem arises when dealing with coding schemes with embedded content-based functionalities. Such schemes have to transmit the time evolution of the shape of the objects on which functionalities are to be applied [4]. In the simplest case, these shapes can be represented as binary images: partitions with only two regions (concept of Video Object Plane -VOP-)

In the case of intra-frame partition coding, several methods, both lossless and lossy, have been proposed [10]. Up

This work has been partially supported by the European Community through the MOMUSYS project of the ACTS program and by the Spanish Goverment: TIC 95-1022-C05-05 to the authors knowledge, among the lossless coding approaches, the lowest coding costs are obtained using extensions of the classical Chain Code [7]. This approach yields average coding costs of 1.34 bits per pixel of contour. This figure accounts for the encoding of both the shape and location of regions in the partition. Nevertheless, if unnoticeable losses are permitted, lossy coding approaches can further reduce this figure without decreasing the visual quality.

The method proposed in [7] has been used as basis for inter-frame partition coding schemes $[2,9,6]$. These techniques perform a prediction of the current partition by motion compensating the previous partition. The prediction error is then simplified and transmitted jointly with the motion parameters.

The main difference between these techniques is the approach that is used to predict the partition. In [2] and [6] the motion parameters are computed using partition information whereas in [9] the information used for estimating the motion is mainly the texture of the images. In these techniques, the prediction error is simplified. This simplification removes small components from the prediction error so that the current partition is coded with losses. Since some losses are allowed in the coding scheme, the efficiency of the technique can be improved by using a lossy coding approach for coding the prediction error, instead of a lossless approach.

This paper presents an intra-frame lossy coding technique for image partitions based on the idea presented in [8]; namely, the Multi-Grid Chain Code (MGCC). This method yields almost unnoticeable losses while reducing the average coding cost from 1.34 [7] to 1 bit per pixel of contour. This technique has been adapted to handle the case of inter-frame partition coding, following the approach proposed in [9].

The presentation of this partition coding technique is structured as follows. In Section 3, the need for an hexagonal contour grid for partition representation is discussed. Section 4 deals with the use of the Multi-Grid Chain Code approach for intra-mode partition coding. Section 5 is describes an inter-mode partition coding approach that combines MGCC and partition prediction techniques. Finally, Section 6 presents some conclusions.

\section{HEXAGONAL CONTOUR GRID}

In order to allow the coding of arbitrary partitions (e.g.: partitions with regions without any constrain), partitions have 
to be correctly represented in terms of boundary information. If contours are represented using the original image grid, regions with one-pixel width elongations rise representation problems. In the case of partitions with more than two regions, several partition configurations can result in the same contour representation [7]. In addition, even in the binary case, the representation of one-pixel width elongations in the original image grid does not allow an easy tracking of contours. In order to track such elongations, either the contour elements are visited twice or the one-pixel width elongation is hañdled as an open contour. In the first case, some contour elements are encoded twice and the possibility of moving backwards on a contour has to be taken into account. In the second case, the position of the open contours within the image has to be encoded, since they are referred to as new pieces of contour.

An hexagonal contour grid [7] allows a one-to-one relationship between partitions and their boundary representation. In addition, with an hexagonal contour grid representation, one-pixel width elongations do not result in open contours since the contour grid surrounds the elongation. Therefore, the coding scheme does not need to have special cases in the encoding procedure for open contours.

This contour grid is related to the partition grid as follows: a contour grid site separates each pair of neighbor sites in the partition grid, assuming a 4-connected neighborhood system for the partition grid. This concept is illustrate in the first example of Figure 1, where square and line segment elements represent sites from the partition and contour grids, respectively. A contour grid site is active if its two closest partition grid sites have different labels. An example of the relationship between both grids is shown in the second example of Figure 1.
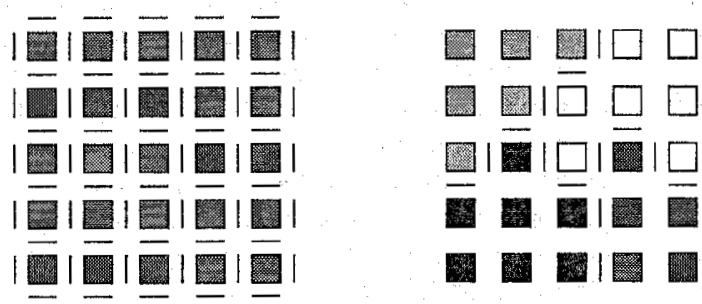

Figure 1: Relationship between partition and contour grid sites.

In this contour grid, the neighborhood system is 6 -connected and, therefore, there are 6 basic movements. Nevertheless, when using derivative chain code in such a grid, the amount of possible movements reduces to 3 as in the 4-connected case: turn right, turn left and straight ahead. Figure 2 compares the possible movements that may appear in both grids.
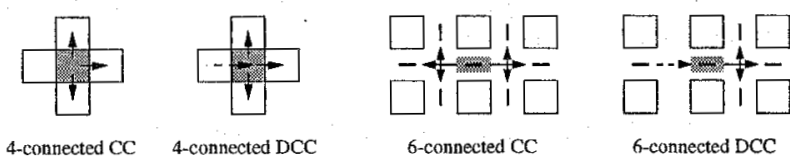

6-connected DCC

Figure 2: Possible movements in 4- and 6-connected grids using $\mathrm{CC}$ and DCC

\section{INTRA-MODE PARTITION CODING USING MULTI-GRID CHAIN CODE}

\subsection{Multi-Grid Chain Code}

Lossy partition coding approaches can be derived from the previous contour image representation. Multi-Grid Chain Code (MGCC) utilizes as basic cell an area of the hexagonal contour grid [1]. This basic cell is shown in Figure 3. A single movement is used to go through the cell. In the example of Figure 3, from the input contour element, marked with 0 , seven possible output contour elements can be reached in order to go through the cell $\{1 \ldots 7\}$. Each one of these output contour elements represents a different movement. If a derivative chain code is used, movements can be represented independently of the input contour element. Symbol 1 corresponds to the contour element which is on the same side as symbol 0 . It defines the rule of symbol assignation, which can be either clockwise or counter-clockwise as can be seen if Figure 3.
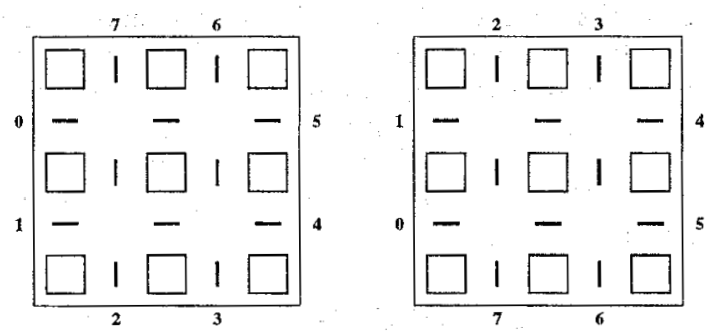

Figure 3: Basic cell of the MGCC

However, a symbol does not only represent a unique contour configuration. As illustrated in Figure 4, a movement (from 0 to 3 in the example) can correspond to two different contour configurations. Therefore, the decoding algorithm has to decide between two possible configurations. Errors in the decision introduce losses in the coding procedure. Note that the only possible error is the region assignment of the central pixel in the basic cell.
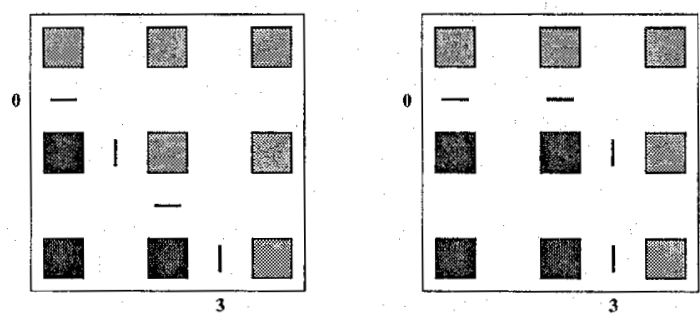

Figure 4: Two possible contour configurations for symbol 3

In the decoder, a special policy has to be followed so that new regions are not created. Although only the label of one pixel can change at each cell in the decoding stage, such changes may split a region into two parts. If a region has an area of one pixel width, the change of label of one of the pixels from this area may strangle the region and divide it into two parts. Such problems are detected and solved in the decoder. 
Some of the symbols in this technique represent larger movements than others. This is the case of symbols 3 or 4 with respect to 1 or 7 . As a consequence, the more often large movements appear, the better the performance of the coding technique. In order to use as many large movements as possible, MGCC changes the position of its basic cells through the grid [8]. This technique combines four grids and, at each position, a cell from one of these grids is selected. This selection relies on the prediction of the contour trajectory at each site. On turn, the prediction assumes boundary smoothness.

An example of the use of the multi-grid approach is presented in Figure 5. In this example, a first contour segment has been coded using the symbol 4 . The following segment of contour would need symbols 7 and 2 to be coded, if a fix grid is used. Nevertheless, when allowing the change of grid, the same segment of contour can be coded using a symbol 4 .

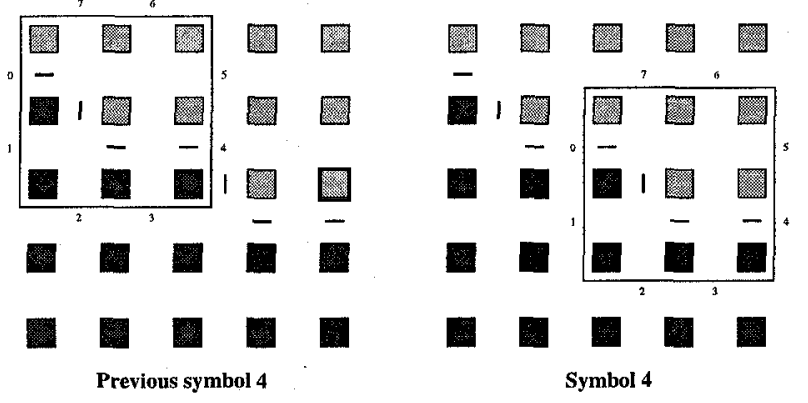

Figure 5: Adaptation of the grid to the previous movement

\subsection{Coding of the region positions}

MGCC is combined with the position coding approach based on the concept of triple points in order to have a complete partition coding technique. In [7], a triple point was defined as a contour element having more than an active neighbor. Therefore, the presence of a triple point is related to the begining of a new contour and can be used to locate regions in the partition. The mark of triple point is directly introduced in the chain of movements. Therefore, the decoder can directly detect the presence and position of a new region [7]. In the case of MGCC, cells having two outputs are marked as being triple points. However, multi-grid cells may have more than two outputs. If a cell presents $k$ different outputs, $k-1$ marks of triple points are introduced in the chain.

\subsection{Intra-mode results}

The use of MGCC results in an average improvement of $25 \%$ on the coding figures with respect to the Chain Code approach [7]. The average bit cost is 1 bit per boundary element, including shape and position information. Furthermore, losses introduced by this technique are almost unnoticeable. This is illustrated in the examples of Figures 6 and 7.

Figure 6 presents the results of encoding the binary shape information of two frames from the sequence Weather. The average number of wrong contour elements after decoding is of $6 \%$ of the total contour elements. Table 1 presents the parameter values from the previous encoding results. Note

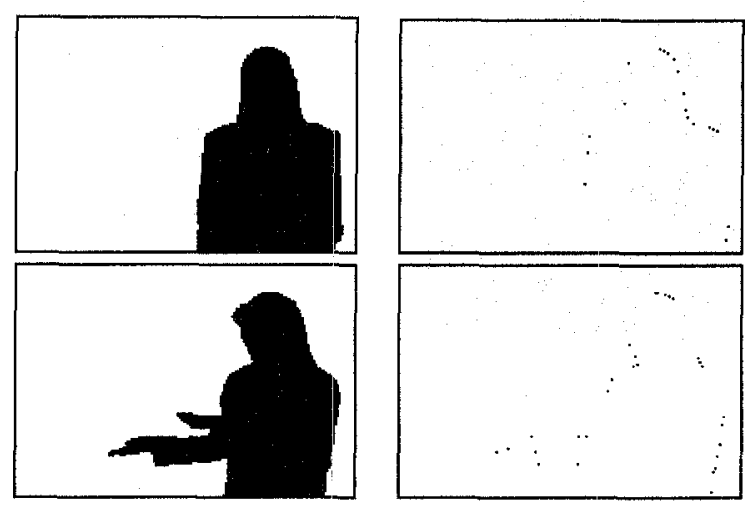

Figure 6: Decoding results of the binary shape information of frames \#10 and \#200 from the sequence Weather and their coding errors.

the reduction from the number of contour elements to the amount of movements used by the MGCC.

\begin{tabular}{|c|c|c|c|c|}
\hline Frame & Size & Cont. Elem. & Wrong CE & MGCC Mov. \\
\hline$\# 10$ & 5796 & 362 & 19 & 162 \\
\hline$\# 200$ & 5683 & 540 & 29 & 237 \\
\hline
\end{tabular}

Table 1: Parameter values from the previous examples

Figure 7 presents the results of encoding a partition of the frame \#0 from the Miss America sequence. The original partition is presented in the first row, whereas the second row presents the decoded partition as well as the coding error. Note that, although the number of erroneous points seems to be high, they are hardly visible in the resulting partition.

\section{INTER-MODE PARTITION CODING}

MGCC can be used in the framework of inter-mode partition coding. As stated in the introduction, MGCC can be combined with a partition prediction technique so that temporal redundancy in the partition sequence is exploited. The proposed inter-mode partition coding algorithm follows the scheme presented in [9]:

1. Partition prediction: The current partition can be predicted using different types of information: previous partitions [6], previous and current partitions [2] or texture information [9].

2. Simplification of the prediction error: An 'overpartition' is defined by merging the predicted partition with the current partition. Each region of the over-partition is analyzed: if the predicted label (number identifying the region) does not correspond to the current label, the region is said to be part of the compensation error. Error regions are eliminated if they are very small or if they do not imply a meaningful grey level difference after coding of its texture. 

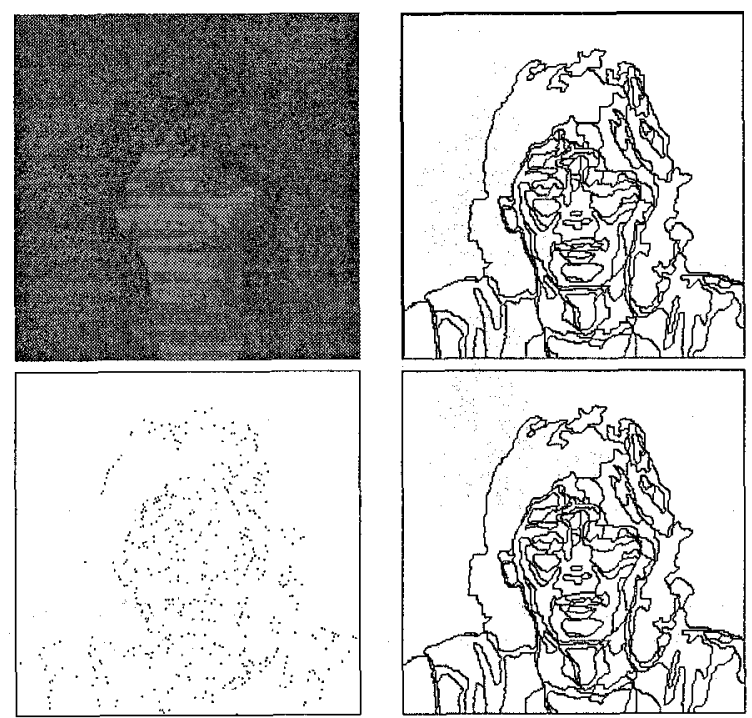

Figure 7: Decoding results of the partition information of a frame from the sequence Miss America.

3. Coding of the error: The error is coded by sending the information necessary to restore the over-partition in the receiver side. The receiver knows the contours of the predicted partition and some extra contours have to be sent. These contours are coded by means of the MGCC approach.

4. Coding of the region label: Once the contours of the partition have been defined, one should assign the correct label to each region. For a large number of regions the correct label is defined by the compensation but for some regions the label should be actually sent.

To code the prediction error, the algorithm of the MGCC has been modified. In the case of coding prediction errors, contour segments cannot be directly located using triple points since the complete partition is not coded at once. On the other hand, a technique that references the starting point of the contour segment with respect to the compensated partition can be used [9].

Figure 8 presents, in the first row, the original shape information from two frames of the sequence Weather. The second row shows the overpartition obtained after motion compensating the previous partition as well as the final coded partition. The areas in dark in the overpartition correspond to the prediction error areas. Some of these areas are directly removed by the simplification step.

\section{CONCLUSIONS}

In this paper, a lossy partition coding technique has been presented. this technique can be applied in both intra- and intermode frame. Results improved that achieved with Chain Code approaches while leading to unnoticeable losses.

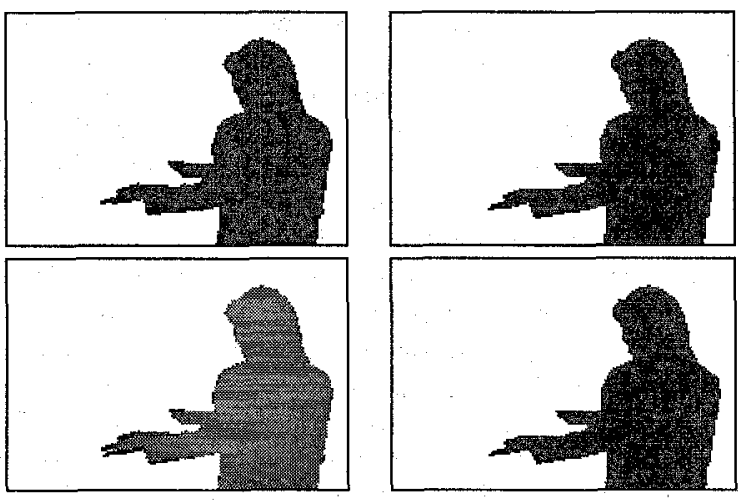

Figure 8: Decoding results of the binary shape information of frame \#202 from the sequence Weather relying on the information of frame \#200.

\section{REFERENCES}

[1] A. Gasull, F. Marqués, and J. A. García. Lossy image contour coding with multiple grid chain code. In Works hop on Image Analysis and Synthesis in Image Coding, page B4, Berlin, Germany, October 1994.

[2] C. Gu. 3D contour image coding by morphological filters and motion estimation. In IEEE, editor, International Conference on Acoustics, Speech and Signal Processing, ICASSP'94, Australia, April 1994.

[3] ISO/IEC JTC1/SC29/WG11. MPEG-4 Proposal Package Description (PPD). July 1995.

[4] ISO/IEC JTC1/SC29/WG11. MPEG-4 Video Verification Model Version 2.0. March 1996.

[5] M. Kunt, A. Ikonomopoulos, and M. Kocher. Second generation image coding techniques. Proceedings of the IEEE, 73(4):549-575, April 1985.

[6] F. Marqués, B. Llorens, and A. Gasull. Partition prediction for partition coding. In EUSIPCO 96, VIII Eu ropean Signal Processing Conference, Trieste, Italy, September 1996.

[7] F. Marqués, J. Sauleda, and A. Gasull. Shape and location coding for contour images. In Picture Coding Symposium, pages 18.6.1-18.6.2, Lausanne, Switzerland, March 1993.

[8] T. Minami and K. Shinohara. Encoding of line drawings with multiple grid chain code. IEEE, Transactions on Pattern Analyis and Machine Intelligence, 8:265-276, March 1986.

[9] P. Salembier. Motion compensated partition coding. In SPIE Visual Communication and Image Processing, VCIP'96, volume 2727, pages 403-415, Orlando, USA, March 1996.

[10] P. Salembier, F. Marqués, and A. Gasull. Coding of partition sequences. In L. Torres and M. Kunt, editors, Video Coding: The second generation approach, pages 125-170. Kluwer Academic Publishers, 1996. 